\title{
PRIME NUMBER THEOREMS FOR THE COEFFICIENTS OF MODULAR FORMS
}

\author{
BY CARLOS JULIO MORENO \\ Communicated by P. T. Bateman, February 25, 1972
}

1. Introduction. The Ramanujan function $\tau(n)$ is defined as the $n$th coefficient in the $q$-expansion of the discriminant function

$$
\Delta(z)=q \prod_{n=1}^{\infty}\left(1-q^{n}\right)^{24}=\sum_{n=1}^{\infty} \tau(n) q^{n}, \quad q=e^{2 \pi i z}, \operatorname{Im}(z)>0 .
$$

It is well known that $\Delta(z)$ spans the space of cusp forms of dimension -12 associated with the unimodular group and is in fact an eigenfunction of the Hecke operators. With $\Delta(z)$ there is associated a Dirichlet series with an Euler product of the type

$$
\varphi(s)=\sum_{n=1}^{\infty} \tau(n) n^{-s}=\prod_{p}\left(1-\tau(p) p^{-s}+p^{11-2 s}\right)^{-1} .
$$

The Dirichlet series $\varphi(s)$ defines a regular function in $\operatorname{Re}(s)>13 / 2$ and satisfies a functional equation of known type. Hardy [3] was the first to observe that the location of the zeros of $\varphi(s)$ gives rise to problems similar to those for the Riemann zeta function $\zeta(s)$. In the Princeton version of [3] Hardy suggested that a prime number theorem for the Ramanujan function

$$
\sum_{p \leqq x} \tau(p) \log p=O\left(x^{13 / 2}\right)
$$

would follow if one could show that $\varphi(s) \neq 0$ for $\operatorname{Re}(s)=13 / 2$. In [6], Rankin settled Hardy's problem by showing that indeed $\varphi(s)$ does not vanish on $\operatorname{Re}(s)=13 / 2$. It is also implicit in Rankin's work that the large $O$ in (3) can be replaced by small $o$. In this note we indicate further improvements on Rankin's result.

2. Statement of results. As in the classical situation of the Riemann zeta function, the following results lead to an improvement of (3).

LEMma 1 (Generalized VON MANGOLDT FORMULA). Let $N_{\varphi}(T)$ denote the number of zeros $\rho=\beta+i \gamma$ of $\varphi(s)$ with $11 / 2 \leqq \beta \leqq 13 / 2$ and $0 \leqq \gamma \leqq T$.

AMS 1970 subject classifications. Primary 10D05, 10H25; Secondary $10 \mathrm{H} 10$.

Key words and phrases. Prime number theorems, Ramanujan function $\tau(n)$, cusp forms, Hecke operators, generalized von Mangoldt formula, explicit formula, eigenfunctions. 
Then

$$
N_{\varphi}(T)=\frac{T}{\pi} \log \frac{T}{2 \pi}-\frac{T}{\pi}+O(\log T) .
$$

LemMa 2 (EXPLiCIT Formula). Let $x$ and $T$ be sufficiently large. Then

$$
\sum_{p \leqq x} \tau(p) \log p=A_{\varphi}(x)-\sum_{|\gamma|<T} x^{\rho} / \rho+R(x, T),
$$

where the second sum runs over those zeros $\rho=\beta+i \gamma$ of $\varphi(s)$ with $11 / 2 \leqq \beta$ $\leqq 13 / 2$ and $|\gamma| \leqq T$. $A_{\varphi}(x)$ and $R(x, T)$ satisfy

$$
A_{\varphi}(x) \ll x^{6} \quad \text { and } \quad R(x, T) \ll \frac{x^{13 / 2}(\log T X)^{2}}{T^{1 / 3}} .
$$

LEMMA 3 (ZERO-FREe REGIONS). The Ramanujan-Dirichlet series is $\varphi(s) \neq 0$ for $s=\sigma+i$ in the region

$$
\sigma \geqq \frac{13}{2}-\frac{B}{\log (|t|+2)},
$$

where $B$ is a suitably chosen positive constant.

MAIN THEOREM.

$$
\sum_{p \leqq x} \tau(p) \log p \ll x^{13 / 2} \exp \left(-A(\log x)^{1 / 2}\right),
$$

where $A$ is a suitably chosen constant.

Remarks. Lemma 1 and Lemma 2 are established in [4] for the more general class of arithmetical functions $\lambda(n)$ which are the coefficients of Dirichlet series whose Mellin transforms are eigenfunctions of the Hecke operators acting on the space of cusp forms of dimension $-k$ associated with the unimodular group. Lemma 3 has also been obtained in this general situation, but the question of whether there is a zero at the real point of the line of absolute convergence has been left open. For the Ramanujan-Dirichlet series $\varphi(s)$, the problem was solved by Rankin [6]. Ogg [5] has shown on the assumption of the Peterson conjecture that there is no such real zero. The Main Theorem also generalizes this but now one must carry an additional term to account for the possibility of there being a real zero of the type described above.

3. Sketch of proofs. Proofs of Lemma 1 can be found in Berndt [1] and Goldstein [2]. The two proofs follow along the classical lines and depend essentially on the functional equation satisfied by $\varphi(s)$ :

$$
(2 \pi)^{-s} \Gamma(s) \varphi(s)=(2 \pi)^{s-12} \Gamma(12-s) \varphi(12-s) .
$$


The proof of Lemma 2 also follows along the classical lines by an application of Perron's Formula to the function

$$
-\frac{\varphi^{\prime}(s)}{\varphi(s)}=\sum_{n=1}^{\infty} \frac{\Lambda_{\varphi}(n)}{n^{s}},
$$

where $\Lambda_{\varphi}(n)$ is the analogue of the von Mangoldt function $\Lambda(n)$ in the theory of the Riemann zeta function. Here one difficulty arises which did not exist in the classical situation, namely we do not know the precise order of magnitude of $\tau(n)$. This difficulty is overcome by using the estimates of Rankin [6] which essentially say that on the average $\tau(n)$ behaves as it should.

The proof of Lemma 3 is an elaboration of Rankin's proof that the Ramanujan-Dirichlet series $\varphi(s)$ does not vanish on the line $\operatorname{Re}(s)=13 / 2$. Rankin's ideas are then complemented with the well-known philosophy that if an analytic function does not vanish at a point, then a suitable open neighborhood of that point can be found where the function also does not vanish. To complete the proof one needs to obtain some estimates for $\varphi(s)$ and its logarithmic derivative near the line of absolute convergence.

The proof of the Main Theorem is a direct consequence of Lemma 2 and Lemma 3 and proceeds along the lines of the classical prime number theorem.

\section{BIBLIOGRAPHY}

1. B. C. Berndt, On the zeros of a class of Dirichlet series. I, II, Illinois J. Math. 14 (1970), 244-258, 678-691. MR 42 \#3261; \#3034.

2. L. Goldstein, A Riemann-von Mangoldt formula for Dirichlet series with functional equations (to appear).

3. G. H. Hardy, Ramanujan, Cambridge Univ. Press, London; Macmillan, New York, 1940. MR 3, 71.

4. C. J. Moreno, Prime number theorems for the coefficients of modular forms and a problem of G. H. Hardy (to appear).

5. A. P. Ogg, On a convolution of L-series, Invent. Math. 7 (1969), 297-312. MR 40 \#88.

6. R. A. Rankin, Contributions to the theory of Ramanujan's function $\tau(n)$ and similar arithmetical functions. I, II, III, Proc. Cambridge Philos. Soc. 35 (1939), 351-372; ibid. 36 (1940), 150-151. MR 1, 69; 203; 400.

The Center for Advanced Study, University of Illinols at Urbana-Champaign, URBANA, ILLINOIS 61801

Current address: DePartment of Mathematics, University of Illinois, Urbana, ILLINOIS 61801 